\title{
A Survey on LMS Context Dimensions for Teacher-Oriented Recommender Systems
}

\author{
http://dx.doi.org/10.3991/ijet.v8i3.2731 \\ I. Bosnić, M. Orlić, M. Žagar \\ University of Zagreb, Zagreb, Croatia
}

\begin{abstract}
In formal education, a majority of e-learning courses is still conducted in a learning management system (LMS). Teachers and authors of educational resources can make use of context-aware recommender systems, which would recommend relevant learning objects to be included in the course. Context data can be obtained, among other sources, from an existing learning environment. This paper describes a two-phase survey of four LMSs - Chamilo, Claroline, Ilias and Moodle - and their characteristics suitable for creating an LMS part of a context model, which would be relevant to implement in recommender systems for this purpose. A sample set of use cases is given, which describes how teachers and content authors could use the system. Based on the survey results, a set of context model dimensions is proposed.
\end{abstract}

Index Terms-context, learning management system, recommender system, content authoring

\section{INTRODUCTION}

For several years, great efforts and breakthroughs in methods of conducting e-learning classes can be observed, such as highly collaborative courses based on social networking, project-based education, Web2.0-based education etc. However, most of the teachers continue to use their preferred LMS and tools they are used to, instead of moving on to new technical solutions [1]. In these cases, even when an existing and well-known environment is used, it is possible to enhance the experience of authoring educational content and teaching, using helper tools available and integrated in the environment. In such applications, the notion of context plays an important role. Some of the main challenges and research topics dealing with context for technology enhanced learning field, as stated by [2], are extending current standards for resources contextualization, automatic acquiring context metadata automatically, understanding contexts, creating tools to help with context-aware applications development, as well as creating context-based activities where users are interacting in a group.

The goal of this paper is to examine which contextual data can be gathered from a conventional learning management system, as an environment for authoring or presenting educational content, to enhance the quality of learning object recommendations to authors of e-learning content. To achieve this, an overview of several open source learning management systems is given, their characteristics - of importance for context modeling - are analyzed, use cases are described and an initial set of possible context dimensions is proposed. It should be noted that the purpose of this paper is not to find the best LMS with regards to potential context characteristics. It is rather to obtain available data which can be used as context dimensions, in defining a part of the context model, with information from an LMS. As not all LMSs contain all data, after defining a model, recommender system implementation can support a subset of proposed characteristics.

The structure of the paper is as follows: Chapter 2 gives an overview of related work. Chapter 3 describes the need for context, focusing on context from a general LMS environment. Chapter 4 presents an overview of selected learning management systems, with an emphasis on available data. It also proposes a set of context dimensions which can be used to specify this part of the model. Chapter 5 describes a sample set of use cases for which such context-aware recommender systems would be useful. Chapter 6 proposes an initial model including LMS context data, while the paper is concluded with Chapter 7 .

\section{RELATED WORK}

An approach to grouping context dimensions in context-aware applications for e-learning, used for content recommendation is described in [4]. Three types of context are defined: knowledge context, social context and technical context.

In an effort to better connect learning management systems with learning object repositories, authors of [3] describe several examples of using context information from an LMS, to describe the content and automatically generate learning object metadata, using LOM specification. For instance, LOM fields such as Main Discipline or Sub Discipline can be inferred from the current category name and course name.

Ontologies as a popular approach to structuring data and relations can be used for various purposes in this field. Course ontology (also called concept hierarchy) which presents course structure and course content outline is discussed in [5]. In course ontology, a term can be the title of a chapter and a section in the course, or a key concept of the course content. Ontologies are also used to describe LMSs and compare common modules in an LMS. In [6], Moodle, Sakai, DotLRN, Claroline and Atutor systems were compared and the structure of an LMS was defined. Another example is using ontologies for modeling several types of context, according to relations among resources, in this case for a learner-centered recommendation system. [7].

Instructional theories, beneficial for authoring learning resources [8], can be modeled using ontologies, as sets of rules for arranging activities and, more important in this case, learning resources in an LMS [9]. These rules formally describe a teaching situation where a learning de- 
sign can be used. As described in [9], such applications can have two goals: testing conformance of a learning design and creating a structure - or even recommending the content - for learning design which is used. It should be stated that such mappings are not complete; only a part of an instructional theory is formally represented. As an example, IMS Learning Design specification is represented using an ontology in [10].

There is a mismatch between the language of learning outcomes on one side, and organization of resources and processes in an LMS, on the other side, as stated in [11]. A framework for capturing intellectual effort (theories, concepts, interactions and constraints), in this case, capturing learning outcomes, identifying their characteristics and mapping such outcomes to Moodle LMS resources or activities, is proposed. In this framework, context is a function of two dimensions: firmness and influence [11].

Various context data, retrieved from an LMS, can be described and classified using taxonomies: they can be defined for task types, task techniques, task interactions, role types, assessment techniques, assessment types, environment types, learning and teaching approach type, outcome type, tool types and resource types. These taxonomies have been used by several teachers in the DialogPlus project for describing their designs [12].

\section{THE NEED FOR CONTEXT}

The popularity of context-aware recommender systems is on the high rise. In some fields context is clearly defined. For instance, in case of pervasive or wearable systems, the context comprises real-world information gathered from sensors [13]. In the field of technology enhanced learning, most recommender systems are intended for use by students/learners. Here, the majority of context information moves from outside world to online environment, with the following groups of context elements, as stated in [14]: computing, location, physical conditions, time, activity, resource, user, social relations. It can be observed that some context characteristics come from physical world, like the current place of learning, e.g. being in a loud environment (public transport) would call for learning objects not requiring full attention of the user. Moving on to online world, context characteristics of student's interacting with resources and LMS, as a part of learning analytics (choosing learning objects to view, downloading, clicking, measuring time spent, etc), is a valuable source of information for recommender systems.

However, when designing a context-aware recommender system which main purpose is recommending learning objects to teachers - more precisely, to authors of e-learning content - for reusing these resources in their own courses, relevant context information differs from information in systems which are used to help students.

There are several factors that need to be taken into consideration when designing such recommendation systems, focused on teachers and reusability:

- focus should be put on formal learning, as the system is mainly intended for e-learning content authors in higher education institutions which tend to be traditional, carrying out content-oriented courses;

- there is a need to keep high quality standards of course resources (both reused and created from scratch), not only in terms of content, but also in structure, pedagogical approaches and presentation;
- the number of teachers authoring a course content in formal learning setting is not high, and the environments are often closed, which reduces the possibility of using collaborative filtering techniques in designing recommender systems;

- on the other hand, it is usual that more than one author work together on creating learning objects, which causes authors to comply with some defined rules regarding the content structure and presentation, to ensure the uniform "look \& feel" of the course content. This can potentially reduce the impact of author's preferences on recommendation algorithm.

Having in mind these and similar issues, context characteristics can be grouped as following:

- content context

- author context

- LMS context

Content context. There are two main sources of content data from which context can be inferred. The first type, which will be used the most, and which comprises the main context part, is a set of dimensions from already existing learning objects in the course. This data can be used throughout the whole recommendation process. The second type, which can be used if recommending is used in the authoring phase (while editing content "on-thefly"), takes into account the information about the resource currently being authored. This provides more context information, especially on the content topic, giving more precise input to the recommendation algorithm, which can result in recommendations more relevant for authoring of the specific resource, whether just for referencing to this recommendation, or reusing it.

This context data is obtained through several characteristics of content. If content metadata is available, it can be used directly, or without extensive processing. Metadata can also be automatically or semi-automatically generated. From the resource, three groups of information can be obtained: structural, pedagogical/instructional and domain.

Author context. Besides learning styles, which are emphasized especially in informal learning, teaching and authoring styles are equally important, especially in formal learning, where a teacher usually has more responsibility for presenting the subject matter. In terms of educational resources, various authors prefer different types of content, or content of the same type with different structure. For instance, overhead slides on the same topic can be created in a variety of flavors: text-descriptive slides, images-only slides, conceptual slides, etc. Authors can have a preferred content creation style, which can be obtained from their previous work, including resources published on Web2.0 sites, such as SlideShare or Prezi. Incorporating such characteristics in author's profile and recommending resources which visually and structurally adhere to teacher's preferences can reduce the amount of work in content authoring and reusing. It should be noted that these systems can be useful for both teachers and content authors, who can be considered as two different types of users. Teachers can profit from such contextaware systems using recommendations for already created content, while authors can use recommendations to improve their authoring process, especially with reusing. 
LMS context. Learning resources do not exist in a „vacuum space“" separately, nor are bundled together in a course without any structure. When educating in a formal setting, a kind of learning environment is used, whether it is a Personal Learning Environment (PLE), Collaborative Learning Environment (CLE) or a more traditional learning management system. Information gathered from such environment, like course categories, structure of course topics and learning objects associated with them, introductions, descriptions, learning goals or learning paths which include navigation information, to name just a few, offer additional contextual information. [15]. Such supplementary information about a learning object can be used to generate the metadata, [3], as well as to ensure a better input for recommendation system.

\section{COMPARISON OF LEARNING MANAGEMENT SYSTEMS}

\section{A. Comparison Setup}

In order to propose a model of contextual information gathered from the learning environment, a set of most popular learning management systems was selected for a two-phase survey. In the first phase, the characteristics and features of these systems were analyzed from both official and unofficial documentation, and also using the official demo sites, which were available for all systems. Emphasis was put on managing the course structure and educational resources. For each system, available and potentially important features for creating an LMS context model were observed. In the second phase of the survey, the systems were tested for availability of all features noted in the first phase and comparison tables were created. A review of approaches to course structuring, from the point of context modeling was made.

For the analysis of systems, official online demo sites were used, as they are usually set up with the default feature set without custom modules, and some offer a demo course on LMS features. Demo sites were running the latest stable versions of learning environments in March 2013.

While testing the environments, the characteristics were grouped in the following categories:

- LMS-level - relevant information at the highest, system level. It consists of data describing course categories and global learning outcomes/goals;

- Course-level - data describing a particular course. Besides the general fields for introduction/title/description and language, course keywords/tags for describing the courses as "folksonomies" can be used in some systems, as well as course comments. Course-level learning outcomes/goals can also be available;

- Structure - internal structure of the course. Most often, the course is divided into learning modules/topics/lessons, or some time-based structure like weeks/months. These structures can range from very simple containers for resources and activities, to multilevel conditional structures - learning paths, including comments and separate learning outcomes;

- Learning objects in LMS - a set of system functionalities and context data related to particular learning objects, but not inherently their part. It can include metadata fields, whether using well-known metadata standards or an excerpt of a metadata set without a formal definition. Resource comments, tags and structure of resource containers - folders - can be used also.

\section{B. Learning Management Systems from Contextual Point of View}

In order to better understand the features of learning environments and results of the survey, a brief description of each LMS is given. The descriptions focus on course structuring and organization of available educational content.

Chamilo is a user-centered and repository-based LMS. Instead of linking resources to courses, content is explicitly linked to a user, who is then considered its owner [16]. Nevertheless, content, which is available in a repository, can be connected to the course, divided into sections, with extensive learning path possibilities available. Metadata support - using Dublin Core specification - is available through the use of external repository, but resources can be publicly commented in a default installation. Chamilo started as a Dokeos/Claroline branch in version 1.x; in version 2. $\mathrm{x}$ it is based on a completely new paradigm, so it is included in this survey together with Claroline.

Claroline LMS organizes content mostly around learning paths, where complete sequences of documents are presented to students. Courses, organized in categories, contain a number of text fields - course description headings - to describe the course in more detail, such as: course description, course content, qualifications and goals, teaching-training activities, support, human and physical resources and methods of evaluation. Custom descriptions are available as well. Besides general information about the content, the files can also be commented. Metadata specifications for resources are not supported.

Moodle is a course oriented LMS. Courses are created in multiple-level categories. Each course consists of sections, either topic- or week-based, which is essentially the same and can be easily swapped; the biggest difference being automatic change of "active section" in week-based course, versus manual change in topic-based course. To be available in the course, all content, resources and activities should be placed in one of the sections. Moodle supports both site-wide learning outcomes in the text-field form and mapping of outcomes to courses and course activities. It also supports conditional activities, which is a way to create learning paths through the available published resources. At the moment, Moodle does not offer native support for metadata specifications; only a few of the metadata fields are available.

Ilias is one of the rare systems to support full LOM metadata specifications, as well as SCORM 2004. It also contains extensive support for creating learning paths, which can be used to implement features like weekly course structure, as in the case of Claroline. Both personal and public comments on learning objects can be written. Tagging is available for both course and learning objects.

\section{Survey Results}

Table 1 shows possible context characteristics at both LMS and course levels, which can be found in particular systems. At LMS-level, in all cases there are multiple-level course categories available. If courses are organized in a domain-oriented way, this can be used to better define the domain topic of a course, with the help of natu- 
ral language processing, If courses are organized in some way important to institutional organization (e.g. using a kind of coding system), but not related to domain of courses or modules, such structure would be hard to use, or even not relevant at all.

Course category descriptions and keywords are not so common. Learning outcomes are usually defined at lower levels, not at the LMS-level. None of the systems contain the feature of similar courses list. It could be possible to define such list from the course categories, depending on the method of structuring categories and more advanced information retrieval algorithms.

At the course-level, more contextual data is available besides course title and introduction, course description, keywords and comments can be used also. Information about pedagogical context can be extracted from learning outcomes or goals, especially if learning outcomes are formally structured (Bloom's taxonomy or Biggs' theory). If they are simply given as free-form text, context obtained in such a way can be less precise, but still relevant. Course language can be used to infer the language of content to be recommended.

Table II shows different ways of organizing course structure inside learning management systems. Usually, a course is divided in parts, called sections, topics or pages, which can sometimes contain notions of sequencing or timing (putting one section after another, whether using exact dates, or just sequentially). More complex organizational structures include learning paths, which can be created explicitly using some learning path specification or content packaging. Alternatively, such structures can be created implicitly using conditional activities and completion features, as implemented in particular LMSs.

Multiple-level sections are partially supported. Section title and description are commonly available, as well as section introduction. Support for keywords/tags per section is inadequate; keywords should be obtained from content available in section and learning objects, using methods already mentioned. Learning outcomes on a section level are not supported in any LMS. Section commenting is almost non-existent.

Table III shows a set of characteristics related to learning objects in LMS. The name and description of a learning object is widely present in systems, as well as some form of comments, whether public or for personal use only. Keywords or tags are often not available, which comes as a surprise, knowing they are generally present in various systems of other types and in metadata specifications, bringing benefits for content analysis. As for widely recognized metadata specifications, such as IEEE LOM or Dublin Core, not many systems support these specifications. For some LMSs, such functionality can be obtained using external plugins. Some metadata fields, like title or description, can certainly be mapped from LMS internal properties, while some other fields can be inferred from the LO itself (e.g. technical metadata), including additional context information, which shows the need for automatic metadata generation methods and tools [17].

\section{USE CASES}

Following the analysis of features in learning management systems, which can be used to model the environ- ment context, we have outlined some of the use cases for a sample of features. Although these use cases can be viewed separately, it is expected that a teacher would want to implement the combination of multiple use cases in his course, so that the final result would provide a better teaching experience.

1. Alice is interested in content recommendations closely related to the domain topic of her course, but mostly general ones. She doesn't strictly expect the resources specialized to a particular course topic, but is open to new content. She helps the recommendation system by filling out the text fields describing the course in general, giving its description and introduction. She would also be interested in getting recommendations for the similar courses. The administrators of her learning environment have set up LMS course categories in a topic-oriented way, so additional information about this and other courses in the same category can be included.

2. Bob would like to get help on authoring the content specific to the current course subtopic. First he needs additional introductory resources which help illustrate the concepts and motivate students, while later on he will prefer more specific and thorough content. To achieve this, he uses an LMS feature of structuring a course - dividing it into topics and describing each topic separately, in addition to metadata and characteristics of already used learning objects. The system will provide him separate recommendations per course topic, whether it is a domain topic, or a structured course part (e.g. course introduction period, course exam period...).

3. Caro is in a similar position as Bob. However, his teaching focuses more on formal learning outcomes, which are described for both the course as a whole, and course subsections, using the Bloom's taxonomy. After annotating the sections-topics with learning outcomes, the recommender system can identify the category of difficulty (knowledge, comprehension, application, analysis, synthesis), and recommend suitable resources.

4. Dave has already worked a lot on a course. He added a number of learning objects, and now he would like to get some recommendations for similar content. A significant help in analyzing the content will be learning object metadata, which can be presented either as just a few fields in LMS, not following any metadata specification, or as a full profile of metadata specification, such as LOM or Dublin Core. Metadata does not have to be specifically defined by the author of already existing content, but can be inferred from the content itself as well.

5. Eve has similar needs as Alice (Use case 1), but is quite interested in the use of tags which can be generated by both students and teachers. Such tags can be matched to vast classification efforts on Web2.0 content providers, popularly called folksonomies. She would like to use course, section and content tagging, and get recommendations for similarly tagged content. 
PAPER

A SURVEY ON LMS CONTEXT DIMENSIONS FOR TEACHER-ORIENTED RECOMMENDER SYSTEMS

TABLE I.

CONTEXT CHARACTERISTICS AT LMS AND COURSE LEVEL

\begin{tabular}{|c|c|c|c|c|}
\hline LMS-level & Chamilo 2.1 & Claroline 1.11 & ILIAS 4.3.2 & Moodle 2.4 \\
\hline Course categories - multiple levels & + & $+^{\mathrm{a}}$ & + & + \\
\hline Course category - title & + & + & + & + \\
\hline Course category - description & - & - & + & + \\
\hline Course category - keywords/tags ${ }^{\mathrm{b}}$ & - & - & + & - \\
\hline Learning outcomes/goals & - & - & - & $+^{\mathrm{c}}$ \\
\hline Similar courses data & - & - & - & - \\
\hline Course-level & Chamilo 2.1 & Claroline 1.11 & ILIAS 4.3 .2 & Moodle 2.4 \\
\hline Course - introduction & + & + & $* \mathrm{~d}$ & $*^{\mathrm{e}}$ \\
\hline Course - title & + & + & + & + \\
\hline Course - description & + & $t^{\mathrm{f}}$ & + & + \\
\hline Course - keywords / tags & - & - & + & $+^{g}$ \\
\hline Learning outcomes / goals & - & $+^{\mathrm{c}}$ & - & $+^{\mathrm{c}}$ \\
\hline Course language & + & + & + & + \\
\hline Course comments & - & + & + & - \\
\hline
\end{tabular}

${ }_{b}^{a}$ One course can be part of more than one, predefined, category

although tags are considered more social than keywords (which are usually used to describe an item officially), in this survey the terms are used interchangeably

learning outcomes are non-structured, text-only fields

can be done as a topic description or start object

can be implemented as a course description

additional text fields: headlines / announcements

$\mathrm{g}$ tags can be added by students

TABLE II.

COURSE STRUCTURE

\begin{tabular}{|c|c|c|c|c|}
\hline Structure & Chamilo 2.1 & Claroline 1.11 & ILIAS 4.3.2 & Moodle 2.4 \\
\hline Type of structure & sections & learning paths & by pages / repository & topics / weeks ${ }^{\text {a }}$ \\
\hline Topics - structure & + & + & $*^{\mathrm{b}}$ & + \\
\hline Topics - multiple levels & - & - & $*^{\mathrm{b}}$ & $*^{* c}$ \\
\hline Topics - introduction & - & $*^{\mathrm{d}}$ & $*^{\mathrm{b}}$ & $*^{\mathrm{d}}$ \\
\hline Topics - title & + & + & $*^{\mathrm{b}}$ & $*^{\mathrm{d}}$ \\
\hline Topics - description & - & + & $* \mathrm{~b}$ & + \\
\hline Topics - keywords / tags & - & - & *b & - \\
\hline Weeks - structure & - & $* \mathrm{~b}$ & *e & + \\
\hline Weeks - multiple levels & - & - & *e & *c \\
\hline Weeks - Introduction & - & $*^{\mathrm{b}}$ & $*^{e}$ & $*^{\mathrm{f}}$ \\
\hline Weeks - title & - & $*^{\mathrm{b}}$ & $*^{\mathrm{e}}$ & $*^{\mathrm{f}}$ \\
\hline \begin{tabular}{|l} 
Weeks - description \\
\end{tabular} & - & + & $*^{\mathrm{e}}$ & $*^{\mathrm{f}}$ \\
\hline Weeks - keywords / tags & - & - & $*^{\mathrm{e}}$ & - \\
\hline Learning outcomes / goals & - & - & - & - \\
\hline Comments - personal & - & - & + & - \\
\hline Comments - public & - & - & - & - \\
\hline Learning paths - sequential & + & + & + & + \\
\hline Learning paths - conditional activities & + & + & + & + \\
\hline
\end{tabular}

Learning paths - conditional activities

weekly structure contains automatic "current week" feature, while "current topic" needs to be set manually

can be implemented as a learning path

can be implemented visually by indenting conten

can be implemented with content availability based on timing

can be implemented as a week description

TABLE III.

LEARNING OBJECTS IN LMS

\begin{tabular}{|l|c|c|c|c|}
\hline \multicolumn{1}{|c|}{ Learning objects in LMS } & Chamilo 2.1 & Claroline 1.11 & ILIAS 4.3.2 & Moodle 2.4 \\
\hline Object - title & + & + & + & + \\
\hline Object - description & + & $-{ }^{\mathrm{a}}$ & + & + \\
\hline Comments - personal & - & - & + & - \\
\hline Comments - public & + & - & $\begin{array}{c}\text { full LOM v1.0, } \\
\text { "quick edit" fields }\end{array}$ & - \\
\hline Metadata formats & - & - & $\begin{array}{c}\text { language, created on, } \\
\text { original URL link }\end{array}$ & - \\
\hline Additional fields & - & - & + & - \\
\hline Tags & + categories & + folders & $\begin{array}{c}\text { + (container objects) } \\
+ \text { repositories }\end{array}$ & + folders \\
\hline $\begin{array}{l}\text { Categories / folders / } \\
\text { Internal repository }\end{array}$ & SCORM 1.2 & $\begin{array}{c}\text { SCORM 1.2 } \\
\text { IMS-CP }\end{array}$ & SCORM 2004 & $\begin{array}{c}\text { SCORM 1.2 } \\
\text { IMS-CP }\end{array}$ \\
\hline Content packaging & & &
\end{tabular}

can be implemented as a public comment

Dublin Core using external repositories via MediaMosa 


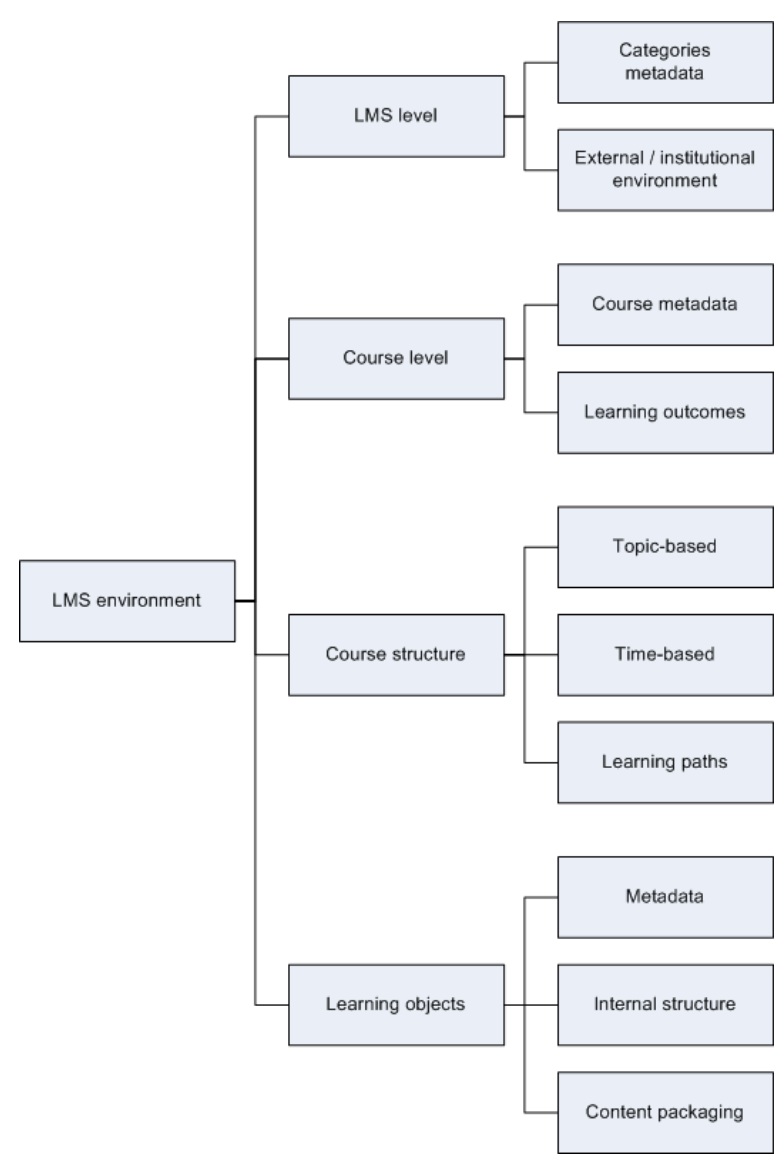

Figure 1. Dimensions of LMS context model

\section{CONTEXT Dimenstions}

Based on the given survey of context dimensions in LMSs, frequency and analysis of particular characteristics and its usability, in Figure 1 we present a set of dimensions which will comprise LMS part of the context model for recommender systems aimed to help content authors.

A subset of characteristics which was included in the survey is not a part of this proposal. There can be different reasons for not including a characteristic in the final proposal: it is not supported by any LMS, it is scarcely supported without probable inclusion in the versions to be published in the near future, or it is estimated that the value of adding this characteristic to the particular context will not be proportional to the effort of inferring or modeling the information. Examples for this are:

- Similar courses data - not available in any learning management systems;

- Learning outcomes at LMS level - these may be too broad to have a relevant impact on recommendations in a particular course, as they describe learning goals from a variety of notably different courses.

\section{CONCLUSION}

While analyzing these context dimensions and use cases, it should be observed that LMS contextual data is only one of three parts comprising the full context model. Context inferred from the author profile and available content (structural, pedagogical, domain) is not a focus of this paper, but plays an important role in usability of use cases. Besides specifically defining proposed dimensions, describing these model subparts is the next step to modeling the complete context for recommender systems for this purpose. In addition to deciding on context dimensions, future work includes a prototype implementation of such model in an LMS, presumably Moodle because of its modularity, popularity, plugin availability and strong developer community. This implementation will serve as a basis for evaluations of context model, recommender system prototype, as well as content provided for recommendations.

\section{REFERENCES}

[1] C. Alario-Hoyos, J. I. Asensio-Pérez, M. L. Bote-Lorenzo, E. Gómez-Sánchez, G. Vega-Gorgojo, and A. Ruiz-Calleja, Integration of external tools in virtual learning environments: main design issues and alternatives, vol. 0. Ieee, 2010, pp. 384-388.

[2] M. Specht and M. Kravcik, "Authoring of learning objects in context," International Journal on ELearning, vol. 5, no. 1, pp. 25-33, 2006.

[3] J. Broisin, P. Vidal, M. Meire, and E. Duval, "Bridging the gap between learning management systems and learning object repositories: exploiting learning context information," in Advanced Industrial Conference on TelecommunicationsService Assurance with Partial and Intermittent Resources ConferenceELearning on Telecommunications Workshop AICTSAPIRELETE05, 2005, pp. 478-483.

[4] Y. Zheng, L. Li, and F. Zheng, "Context-awareness support for content recommendation in e-learning environments," in Information Management Innovation Management and Industrial Engineering 2009 International Conference on, 2009, vol. 3, pp. 514-517.

[5] Q. Zeng, Z. Zhao, and Y. Liang, “Course ontology-based user's knowledge requirement acquisition from behaviors within elearning systems," Computers \& Education, vol. 53, no. 3, pp. 809-818, 2009. http://dx.doi.org/10.1016/j.compedu.2009.04.019

[6] C. E. Montenegro-Marin, J. C. Cueva-Lovelle, O. SanjuanMartinez, and E. R. Neñez-Valdes, "Towards an ontology to describe the taxonomy of common modules in learning management systems," International Jorunal of Interactive Multimedia and Artificial Intelligence, vol. 1, no. SPECIAL ISSUE ON SOFTWARE ENGINEERING, pp. 47-53, 2011.

[7] A. Schmidt and C. Winterhalter, "User context aware delivery of e-learning material: approach and architecture," J UCS, vol. 10, no. 1, pp. 28-36, 2004.

[8] A. F. O'Neal, "The current status of instructional design theories in relation to today's authoring systems," British Journal of Educational Technology, vol. 39, no. 2, pp. 251-267, 2008. http://dx.doi.org/10.1111/j.1467-8535.2008.00815.x

[9] M.-Á. Sicilia, M. D. Lytras, S. Sánchez-Alonso, E. GarcíaBarriocanal, and M. Zapata-Ros, "Modeling instructional-design theories with ontologies: using methods to check, generate and search learning designs," Computers in Human Behavior, vol. 27, no. 4, pp. 1389-1398, 2010. http://dx.doi.org/10.1016/j.chb. 2010.07.040

[10] R. R. Amorim, M. Lama, E. Sánchez, A. Riera, and X. A. Vila, "A learning design ontology based on the ims specification," Educational Technology \& Society, vol. 9, no. 1, pp. 38-57, 2006.

[11] S. Chemboli and C. Boughton, "Contextual course design with omnispective analysis and reasoning," ascilite Conference, 2011.

[12] G. Conole and K. Fill, "A learning design toolkit to create pedagogically effective learning activities," Journal of Interactive Media in Education, vol. 2005, no. 1, pp. 1-16, 2005.

[13] H. Chen, T. Finin, and A. Joshi, "An ontology for context-aware pervasive computing environments," The Knowledge Engineering Review, vol. 18, no. 3, pp. 197-207, 2003. http://dx.doi.org/ $10.1017 / \mathrm{S} 0269888904000025$

[14] K. Verbert et al., "Context-aware recommender systems for learning: a survey and future challenges," IEEE Transactions on Learning Technologies, vol. 6, no. 1, p. 2007, 2012.

[15] X. Ochoa, K. Cardinaels, M. Meire, and E. Duval, "Frameworks for the automatic indexation of learning management systems content into learning object repositories," in World Conference on 
PAPER

A SURVEY ON LMS CONTEXT DIMENSIONS FOR TEACHER-ORIENTED RECOMMENDER SySTEMS

Educational Multimedia, Hypermedia and Telecommunications, vol. 2005, no. 1, pp. 1407-1414.

[16] J.-M. Maes, "Chamilo 2.0: a second generation open source elearning and collaboration platform," International Journal of Advanced Corporate Learning iJAC, vol. 3, no. 3, pp. 26-31,

[17] M. Meire, X. Ochoa, and E. Duval, "Samgi: automatic metadata generation v2.0," in Proceedings of EdMedia07 World Conference on Educational Multimedia Hypermedia and Telecommunications 2007, 2007, pp. 1195-1204

\section{AUTHORS}

I. Bosnić is with the University of Zagreb, Faculty of Electrical Engineering and Computing, Zagreb, Croatia (e-mail: ivana.bosnic@fer.hr).
M. Orlić is with the University of Zagreb, Faculty of Electrical Engineering and Computing, Zagreb, Croatia (e-mail: marin.orlic@fer.hr)

M. Žagar is with the University of Zagreb, Faculty of Electrical Engineering and Computing, Zagreb, Croatia (e-mail: mario.zagar@fer.hr)

This work is supported by the Croatian Ministry of Science, Education and Sport under research project "Software Engineering in Ubiquitous Computing". Submitted, May, 3, 2013. Published as resubmitted by the authors on May, 21, 2013. 